\title{
EMBEDDED CURVES AND FOLIATIONS
}

\author{
HOSSEIN MOVASATI AND PAULO SAD
}

\begin{abstract}
We prove the existence of regular foliations with a prescribed tangency divisor in neighborhoods of negatively embedded holomorphic curves; this is related to a linearization theorem due to Grauert. We give also examples of neighborhoods which can not be linearized.
\end{abstract}

We consider in this paper the problem of finding regular holomorphic foliations in neighborhoods of smooth, compact, holomorphic curves embedded in complex surfaces. More precisely, we fix a positive divisor of a curve and ask whether there exists a holomorphic foliation whose divisor of tangencies with the curve is exactly that divisor. Let us state our main result:

Theorem. Let $C \hookrightarrow S$ be an embedding of the curve $C$ into the surface $S$ such that $C \cdot C<0$.

- if $C \cdot C<4-4 g$, there exists a regular foliation defined in a neighborhood of $C$ and transverse to $C$.

- let a divisor $D=\sum_{k=1}^{l} n_{k} p_{k}$ be given in $C$, with $n_{k} \in \mathbb{N}_{>0}$. If

$$
C \cdot C<4-4 g+\sum_{k=1}^{l}\left(n_{k}-1\right)
$$

there exists a regular foliation $\mathcal{F}$ defined in a neighborhood of $C$ which is transversal to $C$ except at the points $p_{1}, \cdots, p_{l} \in C$, where $\operatorname{tang}_{p_{k}}(\mathcal{F}, C)=n_{k}$ for every $k=1, \ldots, l$.

In the statement $C \cdot C$ stands for the self-intersection number of $C$ in $S$ or, equivalently, the Chern class of the normal bundle of $C$ in $S$; the number $g$ is the genus of $C$. Each number $\operatorname{tang}_{p_{k}}(\mathcal{F}, C)$ is the order of tangency at $p_{k} \in C$ between $C$ and the leaf of $\mathcal{F}$ that passes through the point $p_{k}$.

Our method to prove this theorem consists in i) find a holomorphic line field defined along the curve $C$ with the prescribed set of tangencies 
and the prescribed order of tangencies; for this purpose we have no need to assume that the curve is negatively embedded; ii) extend the line field to a neighborhood of the curve; here we must work under the hypothesis $C \cdot C<0$ in order to assure the annihilation of some cohomology groups.

We should mention that our primary motivation stems from a Linearization Theorem due to Grauert([3]): a curve possesses a neighborhood diffeomorphic to a neighborhood of the zero section of its normal bundle if the embedding is sufficiently negative $(C \cdot C<\min \{0,4-4 g\})$. A proof can be done in two steps. We start by guaranteeing the existence of a foliation transverse to the curve; this is the first case of our Theorem. Once this is acomplished the rest of the proof goes as in ([1])by finding another holomorphic foliation in a neighborhood $V$ of $C$ which has $C$ as a leaf; this foliation and the transverse one are used as a kind of coordinate system for $V$ when the desired diffeomorphism is constructed.

In this paper we discuss also how to produce examples of embeddings such that there are no foliations with a given divisor of tangencies when the negativity condition is violated. In particular, examples where linearization is not possible are presented. All these examples depend on properties of line fields defined along the curve.

\section{Line Fields and Embeddings}

Let us consider an embedding $C \hookrightarrow S$ of the compact, smooth, holomorphic curve $C$ into the surface $S$. In this Section we study existence of line fields defined along $C$; we do not assume $C \cdot C<0$. Existence of a line field with a given divisor of tangencies is always granted when the degree of the divisor is sufficiently bigger then $C \cdot C$. On the other hand, uniqueness (but perhaps not the existence) follows when this degree is not too big, and we will see later how this leads to the construction of interesting examples.

A holomorphic subbundle $Y \hookrightarrow T S \mid C$ is a holomorphic line field along $C$. Equivalently we may say that a line field is a section of the $\mathbb{P}^{1}$-bundle $\mathbb{P}(T S \mid C)$ over $C$. $Y$ has a tangency with $C$ at the point $p \in C$ when the morphism of line bundles $Y \rightarrow N C=\frac{T S \mid C}{T C}$ has a zero at $p$; the order of the zero is the order of tangency between $Y$ and $C$. We write the set of tangencies as an effective divisor $D=\sum_{k=1}^{l} n_{k} D_{k}$ of $C$; the point $p_{k}$ is a point of tangency of order $n_{k}$. 
In order to motivate the next Proposition, let us remark that when $Y$ is a line field along $C$ whose divisor of tangencies with $C$ is $D$ then $Y \simeq \mathcal{O}(-D) \otimes N C$ as line bundles. In fact, the morphism $Y \rightarrow N C$ seen as a section of $H^{0}\left(C, Y^{*} \otimes N C\right)$ has $D$ as its divisor of zeroes; therefore $Y^{*} \otimes N C \simeq \mathcal{O}(D)$. This allows us to confound a line field along $C$ having $D$ as divisor of tangencies with an injective morphism $\mathcal{O}(-D) \otimes N C \rightarrow T S \mid C$. We will from now use $c(N C)$ to denote the Chern class of the normal bundle $N C$ of $C$ in $S$; it is well know that $C \cdot C=c(N C)$.

Proposition 1. Let $D$ be an effective divisor of $C$, and assume

$$
C \cdot C<4-4 g+\sum_{k=1}^{l}\left(n_{k}-1\right)
$$

There exists an injective bundle morphism $Y:\left.\mathcal{O}(-D) \otimes N C \rightarrow T S\right|_{C}$ which has $D$ as divisor of tangencies with $C$.

Proof. Let us use $L:=\mathcal{O}(-D) \otimes N C$ for simplicity. Firstly we construct $Y$ locally, that is, in the restriction of the line bundle $L$ to a small open set $U \subset C$ where it is isomorphic to $U \times \mathbb{C}$. More precisely, we have i) an open subset $U^{\prime}$ of $S$ with coordinates $\left(z_{1}, z_{2}\right) \in \mathbb{C} \times \mathbb{C}$ such that $U=U^{\prime} \cap C$ is $\left\{z_{2}=0\right\}$; ii) a holomorphic function $f$ of $U$ such $\left.D\right|_{U}=\{f=0\}$ and iii) trivialization coordinates $\left(z_{1}, t\right)$ for $\left.L\right|_{U}$; then we may define

$$
Y\left(z_{1}\right)(t)=\left(z_{1}, t, f\left(z_{1}\right) t\right) .
$$

This can be done in each set of an open covering $\left\{U_{i}\right\}_{i \in I}$ of $C$, so we get morphisms $Y_{i}: L\left|U_{i} \rightarrow T S\right| U_{i}$ with the desired property; we assume that the support of each $\left.D\right|_{U_{i}}$ consists of a point at most and that there are no points of tangency in the intersections $U_{i} \cap U_{j}$ when $i \neq j$. Let $\tilde{Y}_{i}$ denote the composition $L\left|U_{i} \rightarrow T S\right| U_{i} \rightarrow N C \mid U_{i}$. As $\tilde{Y}_{i}=a_{i j} \tilde{Y}_{j}$, where $\left\{a_{i j}\right\} \in H^{1}\left(C, \mathcal{O}^{*}(C)\right)$ defines a line bundle $J,\left\{\tilde{Y}_{i}\right\}$ is a section of $J \otimes \operatorname{Hom}(L, N C) \simeq J \otimes L^{*} \otimes N C$ having $D$ as divisor of zeroes, so that $J \otimes L^{*} \otimes N C \simeq \mathcal{O}(D)$. Consequently $J$ is the trivial line bundle and we may suppose $a_{i j}=1$, or $\tilde{Y}_{i}=\tilde{Y}_{j}$.

Now we have that

$$
\left\{Y_{i j}\right\}:=\left\{Y_{i}-Y_{j}\right\} \in H^{1}(C, H o m(L, T C)) \simeq H^{1}\left(C, L^{*} \otimes T C\right) ;
$$

Let $\tilde{D}=\sum_{k=1}^{l} p_{k}$ and $s=\left\{s_{i}\right\} \in H^{0}(C, \mathcal{O}(\tilde{D}))$ whose divisor of zeroes is $\tilde{D}$. Therefore

$$
\left(Y_{i}-Y_{j}\right) \otimes s^{-1} \in H^{1}\left(C, \mathcal{O}(-\tilde{D}) \otimes L^{*} \otimes T C\right)
$$


and by Serre's duality

$$
H^{1}\left(C, \mathcal{O}(-\tilde{D}) \otimes L^{*} \otimes T C\right) \simeq H^{0}\left(C, K C^{2} \otimes \mathcal{O}(\tilde{D}) \otimes \mathcal{O}(-D) \otimes N C\right)
$$

$(K C$ stands for the canonical bundle of $C$ ). By hypothesis the Chern class of the line bundle $K C^{2} \otimes \mathcal{O}(\tilde{D}) \otimes \mathcal{O}(-D) \otimes N C$ is negative; we conclude that $\left(Y_{i}-Y_{j}\right) \otimes s^{-1}=X_{i}-X_{j}$ for $X_{i} \in H^{0}\left(U_{i}, \mathcal{O}(-\tilde{D}) \otimes L^{*} \otimes\right.$ $T C)$, and therefore $Y_{i}-Y_{j}=\left(X_{i}-X_{j}\right) \otimes s=s_{i} X_{i}-s_{j} X_{j}$. We define $Y:=Y_{i}-s_{i} X_{i}$ in each $U_{i}$. Clearly $Y$ is injective outside the support of $\tilde{D}$; at each $p_{i}$, it is equal to $Y_{i}$, so it is also injective. As for the order of tangency at a point $p_{i}$, it coincides with the order of tangency of $Y_{i}$, which is $n_{i}$ by construction.

Consequently, there exists always a holomorphic line field along any curve if we admit a number of tangencies sufficiently big. We see also that there exists always a holomorphic line field with any number of tangencies if $C \cdot C<4-4 g$.

Proposition 2. Assume that $C \cdot C<4-4 g$. There exists an injective bundle morphism $Y:\left.N C \rightarrow T S\right|_{C}$ which has no tangencies with $C$.

Proof. We just have to repeat the arguments applied above without the presence of tangencies. We see that the condition in the statement implies that $Y_{i}-Y_{j}=X_{i}-X_{j}$ for $X_{i} \in H^{0}\left(U_{i}, N C^{*} \otimes T C\right)$.

In the next section we will analyse how to extend this holomorphic line field to a neighborhood of the curve. For the moment, let us state a general result concerning uniqueness.

Proposition 3. Let $D$ be an effective divisor of $C$ and assume

$$
c(N C)>2-2 g+\sum n_{i}
$$

There exists at most one line field along $C$ having $D$ as divisor of tangencies.

Proof. Let us consider two such line fields $Y_{1}$ and $Y_{2}$ as bundle morphisms from $\mathcal{O}(-D) \otimes N C$ into $\left.T S\right|_{C}$. The induced morphisms $\tilde{Y}_{i}$ : $\mathcal{O}(-D) \otimes N C \rightarrow N C$ seen as sections of $\mathcal{O}(D) \otimes N C^{*} \otimes N C=\mathcal{O}(D)$ have the same divisor $D$ of zeroes, so that $\tilde{Y}_{1}=a \tilde{Y}_{2}$ for some $a \in \mathbb{C}^{*}$. It follows that $Y_{1}-a Y_{2}$ is a bundle morphism from $\mathcal{O}(-D) \otimes N C$ to $T C$; the hypothesis tells us that $\mathcal{O}(D) \otimes N C^{*} \otimes T C$ is a negative line bundle and so $Y_{1}-a Y_{2}=0$. 


\section{Neighborhoods of Negatively Embedded Curves}

Before proving the Theorem stated in the Introduction, we collect some properties due to Grauer that are verified in the case of a negatively embedded curve $C \hookrightarrow S([2,[3])$.

- $C$ has a fundamental system of strictly pseudoconvex neighborhoods in $S$.

- if $\mathcal{G}$ is a coherent sheaf defined in one of these neighborhoods, say $V$, and $\mathcal{I}_{C}$ is the ideal sheaf of $C$ in $V$ then

$$
\exists k>0 \text { such that } H^{i}\left(V, \mathcal{I}_{C}^{k} \cdot \mathcal{G}\right)=0, \quad i=1,2 .
$$

Lemma 1. We have $H^{2}\left(V, \mathcal{I}_{C} \cdot \mathcal{G}\right)=0$. Moreover if

$$
H^{0}\left(C, K C \otimes N C^{\nu} \otimes \mathcal{G}^{*} \mid C\right)=0
$$

for all $\nu \geq 1$ then $H^{1}\left(V, \mathcal{I}_{C} \cdot \mathcal{G}\right)=0$.

Proof. From $H^{i}\left(V, \mathcal{I}_{C}^{\nu} / \mathcal{I}_{C}^{\nu+1} \cdot \mathcal{G}\right) \simeq H^{i}\left(C,\left(N C^{*}\right)^{\nu} \otimes \mathcal{G} \mid C\right)$ we get immediately $H^{2}\left(V, \mathcal{I}_{C}^{\nu} / \mathcal{I}_{C}^{\nu+1} \cdot \mathcal{G}\right)=0$. As

$$
H^{1}\left(C,\left(N C^{*}\right)^{\nu} \otimes \mathcal{G} \mid C\right) \simeq H^{0}\left(C, K C \otimes N C^{\nu} \otimes \mathcal{G}^{*} \mid C\right)
$$

(by Serre's duality) we get $H^{1}\left(V, \mathcal{I}_{C}^{\nu} / \mathcal{I}_{C}^{\nu+1} \cdot \mathcal{G}\right)=0$ as well.

Let us consider the short exact sequence

$$
0 \rightarrow \mathcal{I}_{C}^{\nu+1} \cdot \mathcal{G} \rightarrow \mathcal{I}_{C}^{\nu} \cdot \mathcal{G} \rightarrow \mathcal{I}_{C}^{\nu} / \mathcal{I}_{C}^{\nu+1} \cdot \mathcal{G} \rightarrow 0
$$

which leads to

$$
\cdots \rightarrow H^{i}\left(V, \mathcal{I}_{C}^{\nu+1} \cdot \mathcal{G}\right) \rightarrow H^{i}\left(V, \mathcal{I}_{C}^{\nu} \cdot \mathcal{G}\right) \rightarrow H^{i}\left(V, \mathcal{I}_{C}^{\nu} / \mathcal{I}_{C}^{\nu+1} \cdot \mathcal{G}\right) \rightarrow \cdots
$$

Therefore the maps $H^{i}\left(V, \mathcal{I}_{C}^{\nu+1} \cdot \mathcal{G}\right) \rightarrow H^{i}\left(V, \mathcal{I}_{C}^{\nu} \cdot \mathcal{G}\right), \quad i=1,2$, are always surjective. Consequently $H^{i}\left(V, \mathcal{I}_{C}^{k} \cdot \mathcal{G}\right)=0$ for some $k>0$ implies $H^{i}\left(V, \mathcal{I}_{C} \cdot \mathcal{G}\right)=0, i=1,2$.

The next Lemma allows us to extend any line bundle over $C$ to a line bundle over $V$. Of course there are certain line bundles which are extendible regardless of the negativity of the embedding $C \hookrightarrow V$. For example, $K C=K V|C \otimes N C=K V| C \otimes[C] \mid C$, so that $K C$ always has an extension to $V$. Below in our Theorem we find this situation when no tangencies are present.

Lemma 2. The restriction $H^{1}\left(V, \mathcal{O}_{V}^{*}\right) \rightarrow H^{1}\left(C, \mathcal{O}_{C}^{*}\right)$ is surjective.

Proof. Let $J$ be the subsheaf of $\mathcal{O}_{V}^{*}$ defined as

- $J_{q}=\left(\mathcal{O}_{V}^{*}\right)_{q}$ if $q \notin C$. 
- $J_{q}=\left\{\phi \in\left(\mathcal{O}_{V}^{*}\right)_{q} ;\left.\phi\right|_{C} \simeq 1\right\}$ if $q \in C$.

We have then the short exact sequence

$$
1 \rightarrow J \rightarrow \mathcal{O}_{V}^{*} \rightarrow \mathcal{O}_{V}^{*} / J \rightarrow 1
$$

we remark that $\mathcal{O}_{V}^{*} / J$ can be taken as $\mathcal{O}_{C}^{*}$.

In order to have the surjectivity stated above, we need $H^{2}(V, J)=0$. Since the exponencial map gives an isomorphism between $\mathcal{I}_{C}$ and $J$, it is enough to have $H^{2}\left(V, \mathcal{I}_{C}\right)=0$.

\section{Constructing Foliations}

We are able now to prove the Theorem stated in the Introduction.

Let $Y:\left.C \rightarrow T S\right|_{C}$ be the line field constructed in Corollary 1. Let $\left\{U_{i}\right\}$ be a covering of $C$ and $\tilde{U}_{i}$ be an open set such that $\tilde{U}_{i} \cap C=U_{i}$. In each $\tilde{U}_{i}$ we choose a 1 -form $\omega_{i}$ satisfying $\operatorname{ker}\left(\omega_{i}(p)\right)=Y(p)$ when $p \in U_{i}$. We may take coordinates $\left(x_{i}, y_{i}\right) \in \tilde{U}_{i}$ as to have $U_{i}=\left\{y_{i}=0\right\}$ and $\omega_{i}=d y_{i}-x_{i}^{n_{i}} d x_{i}$ (remember that the possibility $n_{i}=0$ is allowed). We remark that $\left.\omega_{i}\right|_{U_{i} \cap U_{j}}=\left.f_{i j} \omega_{j}\right|_{U_{i} \cap U_{j}}$ whenever $U_{i} \cap U_{j} \neq \emptyset, \quad f_{i j} \in$ $Z^{1}\left(\left\{U_{i}\right\}, \mathcal{O}_{C}^{*}\right)$. We denote by $L=\left\{F_{i j}\right\}$ the line bundle over $V$ whose restriction to $C$ is defined by the transition functions $\left\{f_{i j}\right\}$ (Lemma 2); we have

$$
L \mid C=\mathcal{O}(D) \otimes K C^{*},
$$

where $D=\sum_{i=1}^{l} n_{i} p_{i}$. The boundary $\delta\left\{\omega_{i}\right\}$ computed in $Z^{1}\left(S, \Omega_{S}^{1} \otimes L\right)$ belongs effectively to $Z^{1}\left(S, \mathcal{I}_{C} \cdot \Omega_{S}^{1} \otimes L\right)$, where $\Omega_{S}^{1}$ is the sheaf of germs of holomorphic 1-forms of $S$.

We claim that $H^{1}\left(S, \mathcal{I}_{C} \cdot \Omega_{S}^{1} \otimes L\right)=0$. As discussed before, we need that $\forall \nu \geq 1$

$$
H^{0}\left(C, K C \otimes N C^{\nu} \otimes\left(\Omega_{S}^{1} \otimes L\right)^{*} \mid C\right)=0
$$

which depends on

$$
H^{0}\left(C, K C^{2} \otimes N C^{\nu} \otimes \mathcal{O}(-D) \otimes T C\right)=0 \forall \nu \geq 1
$$

and

$$
H^{0}\left(C, K C^{2} \otimes N C^{\nu} \otimes \mathcal{O}(-D) \otimes N C\right)=0 \forall \nu \geq 1
$$

both equalities are true since the Chern classes of the line bundles $K C^{2} \otimes N C^{\nu} \otimes \mathcal{O}(-D) \otimes T C$ and $K C^{2} \otimes N C^{\nu} \otimes \mathcal{O}(-D) \otimes N C$ are negative due to the hypothesis.

It follows that there exists a 0 -cocycle $\left\{\eta_{i}\right\} \in H^{0}\left(\tilde{U}_{i}, \mathcal{I}_{C} \cdot \Omega_{S}^{1} \otimes L\right)=0$ such that 


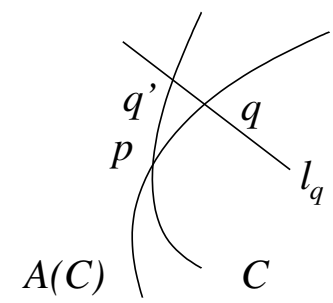

FiguRe 1.

$$
\omega_{i}-F_{i j} \omega_{j}=\eta_{i}-F_{i j} \eta_{j}
$$

and the foliation we look for is defined by the 1-form

$$
\left\{\omega_{i}-f_{i j} \eta_{i}\right\} \in H^{0}\left(V, \Omega_{S}^{1} \otimes L\right) .
$$

Corollary 1. Let $C \hookrightarrow S$ be an embedding of the curve $C$ into the surface $S$ such that $C \cdot C<0$. Then there exists a regular holomorphic foliation defined in a neighborhood of $C$.

\section{Examples}

Example 1. A plane smooth projective curve $C$ different from the projective line does not have a transverse holomorphic line field (this is a particular case of a theorem of Van de Ven ([4])). In fact, suppose $Y$ is a transverse holomorphic line field defined along $C$. We consider a holomorphic automorphism $A$ of the plane close to the Identity which fixes some point $p \in C$ and such that $\left(A_{*} Y\right)(p) \neq Y(p)$; the line field $Y_{A}=A_{*} Y$ is of course transverse to $A(C)$. Given $q \in A(C)$, we denote as $l_{q}$ the projective line tangent to $Y_{A}(q)$ at $q$. We may therefore induce along $C$ a new holomorphic line field $Z \neq Y$ in the following way: given $q \in A(C)$ take $q^{\prime}=l_{q} \cap C$ (the intersection is taken in a small neighborhoood of $C$ ); then $Z\left(q^{\prime}\right)$ is the tangent line to $l_{q}$ at the point $q^{\prime}$, see Figure 1. Since $Z(p)=Y_{A}(p) \neq Y(p)$ and $Z$ is transverse to $C$, we get a contradiction with the Proposition 3 (notice that $c(N C)=d^{2}$ is greater than $3 d-d^{2}=2-2 g$ when $\left.d=\operatorname{degree}(C)>1\right)$.

A different, "foliated" argument goes as follows: suppose that there exists a holomorphic line field transverse to $C$; this is a line bundle $L$ over $C$, as we have seen in Section 1 For each $p \in C$ the line $l(p)$ of $L$ is associated to a projective line $l_{p}$ of the projective plane (this projective line passes through $p$ with direction given by $l(p))$. We take 
some Riemannian metric in $\mathbb{P}^{2}$; since there are neighborhoods of $C$ in $L$ and of $C$ in $\mathbb{P}^{2}$ which are $C^{\infty}$ diffeomorphic (as line bundles), for a small $\eta$ the discs centered at the points of $C$ of radius $\eta$ and contained in the projective lines $\left\{l_{p}\right\}_{p \in C}$ are disjoint, so they form a holomorphic fibration. We pick up a non-constant meromorphic function in $C$ and extend it to a neighborhood of $C$ as a constant along each fiber. This is a meromorphic function that can be extend to all of $\mathbb{P}^{2}$ since the complement of $C$ is a Stein surface. We observe that the extension is constant along each projective line $l_{p}$. The only possibility is that these projective lines form a pencil issued from some point of the plane.

Proposition 3 implies that for a curve with sufficiently positive selfintersection we have at most one transverse holomorphic line field. The above example shows that such a line field may not exist at all. Note that the construction of transverse line fields presented in Proposition 2 is done under the hypothesis that the curve has sufficiently negative self-intersection.

Example 2. The Proposition 3 is useful to get examples of nonexistence of certain regular foliations when the self-intersection of $C$ is not sufficiently negative. In order to see this, let us consider a pair $C \hookrightarrow S$ obtained by the following procedure:

(1) we blow up the origin 0 of the polydisc $\Delta \subset \mathbb{C}^{2}$, introducing an exceptional divisor; we choose the point in this divisor which belongs to the strict transform of $\{y=0\}$ and blow up again. We keep doing this in order to get a chain of projective lines $E_{1}, \ldots, E_{m-1}$ of self-intersection -2 and a last projetive line $E_{m}$ of self-intersection -1 ; there is a holomorphic projection $\pi$ from the resulting surface $\tilde{\Delta}$ to $\Delta$, which collapses $E_{1} \cup \cdots \cup E_{m}$ to 0 , and which is an isomorphism from the complement of this divisor to $\Delta \backslash\{(0,0)\}$. Denote by $q \in E_{m}$ the point which belongs to the strict transform of $\{y=0\}$ and take the $u$ coordinate along $E_{m}$ in order to have $\pi(x, u)=\left(x, u x^{m}\right)$. We take also a polydisc $V=\{x, u) ;|x|<1,|u|<\epsilon\}$, for a small $\epsilon$, around $(x, u)=(0,0)=q \in E_{m}$.

(2) let us consider a line bundle over a compact, holomorphic, smooth curve $\tilde{C}$ whose self-intersection satisfies $\tilde{C} \cdot \tilde{C}>2-2 g$; we select some point in $\tilde{C}$ and introduce coordinates $(\tilde{x}, \tilde{u})$ in a neighborhood $W$ of this point as to have $\{\tilde{x}=$ const $\}$ contained in the linear fiber through $(\tilde{x}, 0) \in \tilde{C}$ for every $\tilde{x}$.

(3) finally we glue $W$ to $V$ by means of a holomorphic diffeomorphism $\Phi: W \rightarrow V$ in order to get a holomorphic surface $\tilde{S}$ 
containing $E_{1} \cup \cdots \cup E_{m} \cup \tilde{C}$ as a divisor whose components have the self-intersection numbers described above; $\Phi$ must send $(\tilde{x}, \tilde{u})=(0,0)$ to $(x, u)=(0,0)=q$, the $\tilde{x}$-axis into the $x$-axis and the $\tilde{u}$-axis transversely to the $u$-axis. We remark that $\tilde{C}$ has a unique field $\tilde{\mathcal{L}}$ of transversal lines because $\tilde{C} \cdot \tilde{C}>2-2 g$; by construction the line $\tilde{\mathcal{L}}_{q}$ is different from $T_{q} E_{m}$.

We blow down $E_{1} \cup \cdots \cup E_{m}$ to $p=(0,0) \in \Delta$ and get a surface $S$ with an embedded curve $C$ such that $C \cdot C>m+2-2 g$ and $p \in C$.

We claim that there exists no regular foliation $\mathcal{F}$ in $S$ transverse to $C \backslash\{p\}$ with order of tangency $0 \leq n \leq m-1$ at $p$. Otherwise after blowing up a times as explained before starting at $p$, we would get a foliation $\tilde{\mathcal{F}}$ transverse to $\tilde{C}$ and having $E_{m}$ as a leaf. Each leaf $\tilde{F}_{s}$ through $s \in \tilde{C}$ has $\tilde{L}_{s}$ as tangent line at $s \in \tilde{C}$; but this property is not verified at the point $q \in \tilde{C} \cap E_{m}$.

We remark that the particular case $m=1$ gives examples of embeddings $C \hookrightarrow S$ such that $C \cdot C>3-2 g$ without transversal foliations to $C$; in particular, there is no neighborhood of $C$ in $S$ which is (holomorphically) diffeomorphic to a neighborhood of $C$ in the total space of its normal bundle.

\section{Plane curves and line fields}

We develop here Example 1 in order to understand the role of tangencies. Let us consider in $\mathbb{P}^{2}$ a smooth algebraic curve $C$ of degree $d$ and a holomorphic line field $X$ along $C$. We have then a holomorphic map $\phi_{X}: C \longrightarrow \check{\mathbb{P}}^{2}$ defined as $\phi_{X}(p)=X(p) \in \check{\mathbb{P}}^{2}$; its image is an algebraic curve $\check{X} \subset \check{\mathbb{P}}^{2}$. Let us denote by $l \in \mathbb{N}$ the degree of $\phi$ as a map from $C$ onto $\check{X}$.

For instance, let us suppose that $X$ is induced by a pencil of lines issued from some point $b \in \mathbb{P}^{2}$. Then $\check{X}$ is a line in $\check{\mathbb{P}}^{2}$ and $\phi_{X}$ has degree $d$ or $d-1$ according to $b \in C$ or $b \notin C$ (in this last case, $X(b)$ is the tangent line to $C$ at $b \in C)$. We have then $\operatorname{tang}(X, C)=d^{2}-d$ or $\operatorname{tang}(X, C)=d^{2}-d-1$.

Proposition 4. $\operatorname{tang}(X, C)=\operatorname{l} \cdot \operatorname{deg}(\check{X})+d^{2}-2 d$.

Proof. We consider $\mathbb{P}\left(\left.T \mathbb{P}^{2}\right|_{C}\right)$, which is a $\mathbb{P}^{1}$-bundle over $C$ with the section $\mathbb{P}(T C)$. The vector bundle $\left.T \mathbb{P}^{2}\right|_{C}$ may be described by the following transition maps:

$$
x_{\alpha}=\xi_{\alpha \beta}\left(z_{\beta}\right) x_{\beta}+\eta_{\alpha \beta}\left(z_{\beta}\right) y_{\beta}, \quad y_{\alpha}=c_{\alpha \beta}\left(z_{\beta}\right) y_{\beta}
$$


where $\left(x_{\beta}, y_{\beta}\right)$ are coordinates for $\left.T \mathbb{P}^{2}\right|_{C}$ at the point of $C$ of coordinate $z_{\beta}, \quad z_{\alpha}=g_{\alpha \beta}\left(z_{\beta}\right), \xi_{\alpha \beta}\left(z_{\beta}\right)=g_{\alpha \beta}^{\prime}\left(z_{\alpha}\right)$ and $\left\{c_{\alpha \beta}\right\}$ defines the normal bundle to $C$ in $\mathbb{P}^{2}$.

In order to get the transition functions of $\mathbb{P}\left(\left.T \mathbb{P}^{2}\right|_{C}\right)$, we put $u_{\beta}=$ $x_{\beta} / y_{\beta}$ and $t_{\beta}=y_{\beta} / x_{\beta}$; then

$$
u_{\alpha}=\frac{\xi_{\alpha \beta}\left(z_{\beta}\right)}{c_{\alpha \beta}\left(z_{\beta}\right)} u_{\beta}+\frac{\eta_{\alpha \beta}\left(z_{\beta}\right)}{c_{\alpha \beta}\left(z_{\beta}\right)}
$$

and

$$
t_{\alpha}=\frac{c_{\alpha \beta}\left(z_{\beta}\right) t_{\beta}}{\xi_{\alpha \beta}\left(z_{\beta}\right)+\eta_{\alpha \beta}\left(z_{\beta}\right) t_{\beta}}
$$

Let us consider the line field $X$ as a section of $\mathbb{P}\left(\left.T \mathbb{P}^{2}\right|_{C}\right)$; we choose also a generic pencil of lines $P$. In the $u$-coodinates, we have

$$
X_{\alpha}=\frac{\xi_{\alpha \beta}\left(z_{\beta}\right)}{c_{\alpha \beta}\left(z_{\beta}\right)} X_{\beta}+\frac{\eta_{\alpha \beta}\left(z_{\beta}\right)}{c_{\alpha \beta}\left(z_{\beta}\right)}
$$

and

$$
P_{\alpha}=\frac{\xi_{\alpha \beta}\left(z_{\beta}\right)}{c_{\alpha \beta}\left(z_{\beta}\right)} P_{\beta}+\frac{\eta_{\alpha \beta}\left(z_{\beta}\right)}{c_{\alpha \beta}\left(z_{\beta}\right)}
$$

The intersection number of both sections $X$ and $P$ with $\mathbb{P}(T C)$ will be denoted by Poles $(X)$ and Poles $(P)$; of course $\operatorname{tang}(X, C)=$ $\operatorname{Poles}(X)$ and Poles $(P)=d^{2}-d$.

¿From the formulae above we see that $\left\{X_{\alpha}-P_{\alpha}\right\}$ is a section of the linear bundle given by the cocycle $\left\{\frac{\xi_{\alpha \beta}\left(z_{\beta}\right)}{c_{\alpha \beta}\left(z_{\beta}\right)}\right\}$, which is $T C \otimes N C^{*}$. Consequently:

$$
\text { Zeroes }(X-P)-\operatorname{Poles}(X-P)=-2 d^{2}+3 d
$$

Therefore Poles $(X)=Z$ eroes $(X-P)-\operatorname{Poles}(P)+2 d^{2}+3 d$. Now since Poles $(P)=d^{2}-d$ and $Z \operatorname{eroes}(X-P)=\operatorname{lddeg}(\check{X})$, we get finally

$$
\operatorname{tang}(X, C)=\operatorname{l} \cdot \operatorname{deg}(\check{X})+d^{2}-2 d \text {. }
$$

Corollary 2. $\operatorname{tang}(X, C) \geq(d-1)^{2}$

This Corollary gives another explanation why a a smooth, plane algebraic curve $C$ of degree greater than one has no transversal holomorphic line field; consequently a neighborhood of $C$ can not be linearized. 
We see also that if we blow up at $d^{2}-2 d$ different points of $C$, the resulting curve $\hat{C}$ has not a linearizable neighborhood as well. In fact, a tranversal holomorphic line field to $\hat{C}$ corresponds to a holomorphic line field along $C$ with at most $d^{2}-2 d$ points of ordinary tangency, which is not possible.

\section{REFERENCES}

[1] C.Camacho, H.Movasati and P.Sad Fibered Neighborhoods of Curves in Surfaces, The Journal of Geometric Analysis, Volume 13, Number 1 (2003), 57-66.

[2] C.Camacho and H.Movasati Neighborhoods of Analytic Varieties, Monografias del IMCA (2003).

[3] H.Grauert, Über Modifikationen and exzeptionelle analytische Mengen, Math. Ann. 146 (1962), 331-368.

[4] A. Van de Ven A property of algebraic varieties in complex projective spaces, Colloque Gom. Diff. Globale (Bruxelles, 1958),151-152, Centre Belge Rech. Math., Louvain 1959. 